\title{
Algebraic Properties of Parikh Matrices of Binary Picture Arrays
}

\author{
Somnath Bera, ${ }^{1}$ Sastha Sriram, ${ }^{2}$ Atulya K. Nagar, ${ }^{3}$ Linqiang Pan $\mathbb{D}^{1}$ \\ and K. G. Subramanian $\left(\mathbb{D}^{3}\right.$ \\ ${ }^{1}$ School of Artificial Intelligence and Automation, Huazhong University of Science and Technology, Luoyu Road 1037\#, \\ Wuhan 430074, Hubei, China \\ ${ }^{2}$ Department of Mathematics, School of Arts, Science and Humanities, SASTRA Deemed University, Tanjore, \\ Tamil Nadu 613 401, India \\ ${ }^{3}$ School of Mathematics, Computer Science and Engineering, Liverpool Hope University, Liverpool L16 9JD, UK
}

Correspondence should be addressed to Linqiang Pan; lqpan@mail.hust.edu.cn and K. G. Subramanian; kgsmani1948@gmail.com

Received 20 April 2020; Accepted 4 June 2020; Published 29 June 2020

Academic Editor: Ali Jaballah

Copyright (C) 2020 Somnath Bera et al. This is an open access article distributed under the Creative Commons Attribution License, which permits unrestricted use, distribution, and reproduction in any medium, provided the original work is properly cited.

A word is a finite sequence of symbols. Parikh matrix of a word is an upper triangular matrix with ones in the main diagonal and nonnegative integers above the main diagonal which are counts of certain scattered subwords in the word. On the other hand, a picture array, which is a rectangular arrangement of symbols, is an extension of the notion of a word to two dimensions. Parikh matrices associated with a picture array have been introduced, and their properties have been studied. Here, we obtain certain algebraic properties of Parikh matrices of binary picture arrays based on the notions of power, fairness, and a restricted shuffle operator extending the corresponding notions studied in the case of words. We also obtain properties of Parikh matrices of arrays formed by certain geometric operations.

\section{Introduction}

"Combinatorics on words" [1] is a comparatively new branch of discrete mathematics with applications in many fields. The work [2] of the Norwegian mathematician Axel Thue (1863-1922) is considered to be the origin for the beginning of this new branch of mathematics. A finite word or simply a word is a finite sequence of symbols in a finite set called an alphabet. The Parikh vector [3] of a finite word, which has played a significant role in the theory of formal languages [3], expresses a numerical property of the word by counting the number of occurrences of the different symbols in the word.

The recently introduced notion of the Parikh matrix [4] of a word over an ordered alphabet is an extension of the Parikh vector. The Parikh matrix of a word, which is based on subwords (also called scattered subwords) of the word, is a very interesting and effective tool in the study of certain numerical properties of the word. Intensive work (see, for example, [5-11]) has taken place in investigating properties of words based on associated Parikh matrices. Such theoretical studies have dealt with problems of great interest related to words such as inequalities on the numbers of occurrences of subwords, injectivity of the mapping involved in defining the Parikh matrix, and other directions [12]. An application of the Parikh matrix in message authentication is considered in [13].

On the other hand, a picture array or simply an array, having a rectangular arrangement of symbols in rows and columns, is an extension of a word to two dimensions (2D) [3]. Several combinatorial properties of arrays have also been intensively investigated [14-20]. For instance, notions such as repetitions of subarrays in 2D arrays are studied in [15-17, 19], while periodicity in arrays is dealt with in $[14,18]$. The notion of the Parikh matrix of a word has been extended to row and column Parikh matrices of picture arrays in [21], and their properties have been studied. The problem of reconstruction of $2 \mathrm{D}$ binary images has been studied [22] based on Parikh matrices. 
Here, we consider binary picture arrays and establish properties of the Parikh matrices of power of an array, fairness of an array, and a restricted shuffle operator on arrays, by extending the corresponding notions [20, 23, 24] investigated in the case of words. We also obtain properties of Parikh matrices of arrays formed by certain geometric operations. A preliminary version of this work was presented in the conference MICOPAM 2018 [25].

\section{Preliminaries}

For notions of formal string language theory and two-dimensional languages, not explained here, the reader is referred to [3]. We recall only some basic notions.

A set $\Sigma$, called an alphabet, is a finite set of symbols. A word $w$ over $\Sigma$ is a finite sequence of symbols over $\Sigma$. The set of all words over $\Sigma$ is denoted by $\Sigma^{*}$, and $\lambda$ is the empty word with no symbols. An alphabet $\Sigma=\left\{a_{1}, a_{2}, \ldots, a_{k}\right\}$, with an order $a_{1}<a_{2}<\cdots<a_{k}$ defined on it, is called an ordered alphabet, and we write $\Sigma=\left\{a_{1}<a_{2}<\cdots<a_{k}\right\}$. A word $u$ is said to be a scattered subword (or simply subword) of a word $w \in \Sigma^{*}$ if there exist words $x_{1}, x_{2}, \ldots, x_{n}, y_{0}, y_{1}, \ldots, y_{n} \in \Sigma^{*}$ (possibly empty) such that $u=x_{1}, x_{2}, \ldots, x_{n}$ and $w=y_{0}$ $x_{1} y_{1}, \ldots, y_{n-1} x_{n} y_{n}$. The length of a word $w \in \Sigma^{*}$, denoted by $|w|$, is the number of symbols present in $w$. The number of occurrences of a word $u$ as a subword of $w$ is denoted by $|w|_{u}$.

A picture array (or simply an array) $A$ over $\Sigma$ of size $m \times n, m, n \geq 1$ is a rectangular arrangement of symbols in $\Sigma$ in $m$ rows and $n$ columns. For example, $\begin{array}{lll}a & b & a \\ b & a & b\end{array}$ is a $2 \times 3$ binary array over the binary alphabet $\Sigma=\{a, b\}$. We denote the set of all $m \times n$ arrays over $\Sigma$ by $\Sigma^{m \times n}$. If $X \in \Sigma^{m \times n}$, we denote by $\left|X_{i}\right|_{x}$, the number of symbol $x$ in the $i^{\text {th }}$ row (or in the $i^{\text {th }}$ column) $X_{i}$ of array $X$, and by $|X|_{x}$, the sum $\sum_{i=1}^{m} X_{i}$. For two arrays $X$ and $Y$ with the same number of rows (resp. columns), the column (resp. rows) catenation $X \circ Y$ (resp. $X \diamond Y$ ) is the array obtained by juxtaposing the array $Y$ on the right (resp. below) of the array $X$.

Throughout the rest of the paper, we consider only a binary ordered alphabet $\Sigma$ and binary arrays over $\Sigma$ unless specified otherwise. We now recall the definition of the Parikh matrix mapping [4] restricting it to a binary alphabet. Let $\mathscr{M}_{3}$ be the monoid of $3 \times 3$ upper triangular matrices with nonnegative integer entries and unit diagonal with respect to the multiplication of matrices. The unit $3 \times 3$ matrix is denoted by $I_{3}$. For a matrix $M \in \mathscr{M}_{3}$, the $(i, j)$ th entry is denoted by $M_{i j}$.

Definition 1 (see [4]). Let $\Sigma=\left\{a_{1}<a_{2}\right\}$ be an ordered alphabet. The Parikh matrix mapping, denoted by $\psi_{3}$, is the morphism: $\psi_{3}: \Sigma^{*} \longrightarrow \mathscr{M}_{3}$ defined as follows: $\psi_{3}(\lambda)=I_{3}$ and for $1 \leq k \leq 2, \psi_{3}\left(a_{k}\right)=\left(M_{i j}\right)_{1 \leq i, j \leq 3}$ where $M_{i i}=1$ for $1 \leq i \leq 3, M_{k(k+1)}=1$, and all other entries are zero. For a word $w=w_{1} w_{2} \cdots w_{n}$ with $w_{i} \in \Sigma$, the Parikh matrix of $w$ is given by $\psi_{3}(w)=\psi_{3}\left(w_{1}\right) \psi_{3}\left(w_{2}\right) \cdots \psi_{3}\left(w_{n}\right)$.

If $M_{1}, M_{2} \in \mathscr{M}_{3}$ are two matrices, then the partial sum $M=M_{1} \oplus M_{2}$ is defined [8] as the usual sum of matrices $M_{1}$ and $M_{2}$ except that the diagonal entries of $M$ by definition have the value 1 .

\section{Row and Column Parikh Matrices of a Binary Picture Array}

The notion of the Parikh matrix of a word has been extended to a picture array in [21] by introducing a row Parikh matrix and column Parikh matrix of an array, which we recall now again restricting to a binary alphabet.

Definition 2. Let $\Sigma=\left\{a_{1}<a_{2}\right\}$ and the array $A \in \Sigma^{m \times n}$. Let the word in the $i^{\text {th }}$ row of $A$ be $x_{i}, 1 \leq i \leq m$, and the vertical word in the $j^{\text {th }}$ column of $A$ be $y_{j}, 1 \leq j \leq n$. Let the Parikh matrices of $x_{i}$ and $y_{j}$ be, respectively, $M\left(x_{i}\right), 1 \leq i \leq m$, and $M\left(y_{j}\right), 1 \leq j \leq n$. Then, the row Parikh matrix $M_{r}(A)$ of $A$ is defined as $M_{r}(A)=M\left(x_{1}\right) \oplus \cdots \oplus M\left(x_{m}\right)$ and the column Parikh matrix $M_{c}(A)$ of $A$ is defined as $M_{c}(A)=M\left(y_{1}\right) \oplus \cdots \oplus M\left(y_{n}\right)$.

As an illustration, consider the array $A=\begin{array}{lll}a & b & a \\ b & a & b\end{array}$. Denoting the words in the rows as $u=a b a$ and $v=b a b$, the row Parikh matrix of $A$ is $M_{r}(A)=M(u) \oplus M(v)=$ $\left(\begin{array}{lll}1 & 2 & 1 \\ 0 & 1 & 1 \\ 0 & 0 & 1\end{array}\right) \oplus\left(\begin{array}{lll}1 & 1 & 1 \\ 0 & 1 & 2 \\ 0 & 0 & 1\end{array}\right)=\left(\begin{array}{lll}1 & 3 & 2 \\ 0 & 1 & 3 \\ 0 & 0 & 1\end{array}\right)$

We first obtain a property of the row (resp. column) Parikh matrix of a binary picture array, extending a corresponding property [8] of the Parikh matrix of a binary word.

Theorem 1. For integers $m, n(\geq 1)$, suppose $M=$ $\left(\begin{array}{lll}1 & r & t \\ 0 & 1 & s \\ 0 & 0 & 1\end{array}\right) \in \mathscr{M}_{3}$. If $M$ is the row (resp. column) Parikh matrix of an $m \times n$ binary array $A$, then $r+s=m n$ and $t \leq n r-\sum_{i=1}^{m} r_{i}^{2} \quad\left(\right.$ resp. $\left.t \leq m r-\sum_{i=1}^{n} c_{i}^{2}\right)$, where $\left|A_{i}\right|_{a}=r_{i}$ $(1 \leq i \leq m)$ (resp. $\left.\left|A_{i}\right|_{a}=c_{i}(1 \leq i \leq n)\right)$ with $A_{i}$ being the $i^{\text {th }}$ row (resp. column) of $A$.

Proof. We prove the result only for the row Parikh matrix as the result for the column Parikh matrix can be proved in a similar manner. Let $M$ be the row Parikh matrix of an $m \times n$ binary array $A$. Then, $A$ has $m n$ symbols, $r a$ 's, and $s b$ 's, so that $r+s=m n$. Let $\left|A_{i}\right|_{a}=r_{i}$ with $A_{i}$ being the $i^{\text {th }}(1 \leq i \leq m)$ row of $A$. Then, $\sum_{i=1}^{m} r_{i}=r$, and the number of $b$ 's in the $i^{\text {th }}$ row is $\left(n-r_{i}\right)$. Therefore, the maximum number of $a b$ 's in the $i^{\text {th }}$ row is $r_{i}\left(n-r_{i}\right)$. Thus, the maximum number of $a b$ 's in the row Parikh matrix of $A$ is $\sum_{i=1}^{m} r_{i}\left(n-r_{i}\right)$ so that $t \leq n r-\sum_{i=1}^{m} r_{i}^{2}$.

Corollary 1. Let $M$ be as in Theorem 1. If $M$ is the row (resp. column) Parikh matrix of an $m \times n$ array, then $r+s=m n$ and $t \leq n r-\left(r^{2} / m\right)$ (resp. $\left.t \leq m r-\left(r^{2} / n\right)\right)$.

This result follows from Theorem 1 by the Cauchy-Schwarz inequality $\sum_{i=1}^{m} r_{i}^{2} \geq(1 / m)\left(\sum_{i=1}^{m} r_{i}\right)^{2}$.

\section{Parikh Matrix of Power of an Array}

The Parikh matrix of a word $w$ raised to an arbitrary power, denoted as $w^{p}$, for an integer $p \geq 1$ has been studied in [23]. 
Here, we consider power of an array which has been introduced in [20].

Definition 3. Let $A$ be an $m \times n$ array. Then, $p \times q$ power of $A$, denoted by $A^{(p \times q)}$, is the $p m \times q n$ picture array such that $A_{i j}^{(p \times q)}=A_{(i \bmod m)(j \bmod n)}$, for all $1 \leq i \leq p m$ and $1 \leq j \leq q n$.

Example 1. Let $A=\begin{array}{lll}a & a & b \\ b & a & b\end{array}$ be a $2 \times 3$ two-dimensional array. The $2 \times 4$ power of $A$ is given by $A^{(2 \times 4)}=$ $\begin{array}{llllllllllll}a & a & b & a & a & b & a & a & b & a & a & b\end{array}$ $\begin{array}{llllllllllll}b & a & b & b & a & b & b & a & b & b & a & b\end{array}$ $\begin{array}{llllllllllll}a & a & b & a & a & b & a & a & b & a & a & b\end{array}$ $\begin{array}{llllllllllll}b & a & b & b & a & b & b & a & b & b & a & b\end{array}$

Theorem 2. Let $M=\left(\begin{array}{lll}1 & r & t \\ 0 & 1 & s \\ 0 & 0 & 1\end{array}\right)$ be the row Parikh matrix of a binary $m \times n$ array $A$ over $\{a<b\}$. Then, the row Parikh matrix of the power $A^{(p \times q)}$ is given by $\left(\begin{array}{ccc}1 & p q r & p q t+(p q(q-1) / 2) \sum_{i=1}^{m} r_{i} \cdot s_{i} \\ 0 & 1 & p q s \\ 0 & 0 & 1\end{array}\right)$, where $\left|A_{i}\right|_{a}=r_{i}$ and $\left|A_{i}\right|_{b}=s_{i}$, with $A_{i}$ being the $i^{\text {th }}$ row of $A$.

Proof. We have $A^{(p \times q)}=\left(A^{(1 \times q)}\right)^{(p \times 1)}$. Now, $A^{(1 \times q)}$ is the column catenation $A_{0} \cdots \circ \mathrm{A}$ of $A$ with itself, $q$ times. Let $r_{i}$, $s_{i}$, and $t_{i}$ denote the number of $a$ 's, $b$ 's, and $a b^{\prime}$ 's in the $i^{\text {th }}$ row $x_{i}(1 \leq i \leq m)$ of $A$. Then, the $i^{\text {th }}$ row of $A^{(1 \times q)}$ is $x_{i}^{q}$. Using the formula in [23] (Theorem 3.1), the Parikh matrix of $x_{i}^{q}$ is given by $\psi_{3}\left(x_{i}^{q}\right)=\left(\begin{array}{ccc}1 & q r_{i} & q t_{i}+(q(q-1) / 2) r_{i} \cdot s_{i} \\ 0 & 1 & q s_{i} \\ 0 & 0 & 1\end{array}\right)$. Therefore, the row Parikh matrix of $A^{(1 \times q)}$ is $=\left(\begin{array}{ccc}1 & q r & q t+(q(q-1) / 2) \sum_{i=1}^{m} r_{i} \cdot s_{i} \\ 0 & 1 & q s \\ 0 & 0 & 1\end{array}\right)$. Since the array $A^{(p \times q)}$ is the row catenation $A^{(1 \times q)} \diamond \cdots \diamond A^{(1 \times q)}$ of the array $A^{(1 \times q)}$ with itself $p$ times, each of the rows of the array $A^{(1 \times q)}$ is repeated $p$ times in the same order in $A^{(p \times q)}$. This means that $\left|A^{(p \times q)}\right|_{a}$ is $p$ times $\left|A^{(1 \times q)}\right|_{a}$, i.e., $\left|A^{(p \times q)}\right|_{a}$ is $p q r$. Likewise, for $b$ 's and $a b$ 's. This proves the required result.

The notion of $M$-ambiguity of words has been extended to two-dimensional picture arrays in [21]. We now recall this.

Definition 4. The arrays $A, B \in \sum^{m \times n}$ are said to be (i) $M$-row equivalent if $M_{r}(A)=M_{r}(B)$ and (ii) $M$-column equivalent if $M_{c}(A)=M_{c}(B)$. The arrays $A$ and $B$ are said to be $M$-equivalent, denoted by $A \equiv_{M} B$, if they are both $M$-row equivalent and $M$-column equivalent. An array $A \in \Sigma^{m \times n}$ is $M$-ambiguous (or simply ambiguous) if it is $M$-equivalent to another distinct array; otherwise, it is unambiguous.

In [23], it is shown that for any two words $v, w \in \Sigma^{*}$, $|\Sigma| \geq 2$, either of the following statements (i)or(ii) holds: (i) $v^{k} \equiv{ }_{M} w^{k}$, for all positive integers $k$; (ii) $v^{k} \not{ }_{M} w^{k}$, for all positive integers $k$. In the case of binary picture arrays, the situation is different as seen from the following proposition.

Proposition 1. There are $M$-row equivalent picture arrays whose powers are not M-row equivalent and conversely.

This proposition is illustrated in the following example.

Example 2. We consider binary arrays $A=\begin{array}{lll}a & a & b \\ b & a & a\end{array}$ and $B=\begin{array}{lll}a & b & b \\ a & a & a\end{array}$. Then, $A^{(1 \times 2)}=\begin{array}{llllll}a & a & b & a & a & b \\ b & a & a & b & a & a\end{array}$ and $B^{(1 \times 2)}=\begin{array}{llllll}a & b & b & a & b & b \\ a & a & a & a & a & a\end{array}$ Now, $M_{r}(A)=M_{r}(B)=$ $\left(\begin{array}{lll}1 & 4 & 2 \\ 0 & 1 & 2 \\ 0 & 0 & 1\end{array}\right)$ so that the binary arrays $A$ and $B$ are $M$-equivalent.

But,

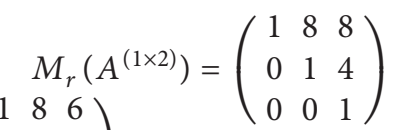
and $M_{r}\left(B^{(1 \times 2)}\right)=\left(\begin{array}{lll}1 & 8 & 6 \\ 0 & 1 & 4 \\ 0 & 0 & 1\end{array}\right) \quad$ so that $M_{r}\left(A^{(1 \times 2)}\right)$ and $M_{r}\left(B^{(1 \times 2)}\right)$ are not $M$-equivalent.

We next consider binary arrays $C=\begin{array}{llll}a & a & b & b \\ b & b & a & a\end{array}$ and $D=\begin{array}{llll}a & a & b & a \\ a & b & b & b\end{array} . \quad$ Then, $\quad \begin{array}{llll}b & b & a & a \\ & & & C^{(1 \times 2)}=\end{array}$ $\begin{array}{llllllll}a & a & b & b & a & a & b & b\end{array}$ $\begin{array}{lllllllll}b & b & a & a & b & b & a & a\end{array}$ $D^{(1 \times 2)}=\begin{array}{llllllll}a & a & b & a & a & a & b & a \\ a & b & b & b & a & b & b & b\end{array}$.

We $\left.\quad \begin{array}{ccc}1 & 4 & 5 \\ 0 & 1 & 4\end{array}\right) \quad M_{r}(C)=\left(\begin{array}{lll}1 & 4 & 4 \\ 0 & 1 & 4 \\ 0 & 0 & 1\end{array}\right) \quad$ and $M_{r}(D)=\left(\begin{array}{lll}1 & 4 & 5 \\ 0 & 1 & 4 \\ 0 & 0 & 1\end{array}\right)$ so that the binary arrays $C$ and $D$ are

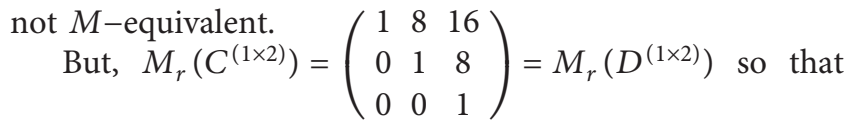
$M_{r}\left(C^{(1 \times 2)}\right)$ and $M_{r}\left(D^{(1 \times 2)}\right)$ are $M$-equivalent.

The next result gives a sufficient condition for two $M$-row equivalent binary picture arrays to have their powers also $M$-row equivalent.

Theorem 3. Let $A$ and $B$ be two $m \times n M$-row equivalent binary arrays over $\Sigma=\{a<b\}$. Then their powers $A^{(p \times q)}$ and $B^{(p \times q)}$ are $M$-row equivalent if $\sum_{i=1}^{m} r_{i}^{2}=\sum_{i=1}^{m} u_{i}^{2}$, where $\left|A_{i}\right|_{a}=r_{i}$ and $\left|B_{i}\right|_{a}=u_{i}, 1 \leq i \leq m$, with $A_{i}$ and $B_{i}$ being the $i^{\text {th }}$ rows of $A$ and $B$, respectively.

Proof. Let $A$ and $B$ be two $m \times n M$-row equivalent binary arrays over $\Sigma=\{a<b\}$ and $M_{r}(A)=M_{r}(B)=\left(\begin{array}{ccc}1 & r & t \\ 0 & 1 & s \\ 0 & 0 & 1\end{array}\right)$.

Then, $\sum_{i=1}^{m} r_{i}=\sum_{i=1}^{m} u_{i}=r$ where $r_{i}$ and $u_{i}, 1 \leq i \leq m$, are the number of $a$ 's in the $i^{\text {th }}$ row of $A$ and $B$, respectively. Also, the number of $b$ 's in the $i^{\text {th }}$ row of $A$ and $B$, respectively, is $\left(n-r_{i}\right)$ and $\left(n-u_{i}\right)$. Suppose $\sum_{i=1}^{m} r_{i}^{2}=\sum_{i=1}^{m} u_{i}^{2}$. Now, using Theorem 2, we have 


$$
\begin{aligned}
M_{r}\left(A^{(p \times q)}\right) & =\left(\begin{array}{ccc}
1 & p q r & \alpha \\
0 & 1 & p q s \\
0 & 0 & 1
\end{array}\right), \\
M_{r}\left(B^{p \times q}\right) & =\left(\begin{array}{ccc}
1 & p q r & \beta \\
0 & 1 & p q s \\
0 & 0 & 1
\end{array}\right),
\end{aligned}
$$

where $\alpha=p q t+(p q(q-1) / 2) \sum_{i=1}^{m} r_{i} \cdot\left(n-r_{i}\right)$ and $\beta=$ $p q t+(p q(q-1) / 2) \sum_{i=1}^{m} u_{i} \cdot\left(n-u_{i}\right)$. We now prove that $\alpha=\beta$ which will complete the proof.

We have

$$
\begin{aligned}
\alpha & =p q t+\frac{p q(q-1)}{2} \sum_{i=1}^{m} r_{i} \cdot\left(n-r_{i}\right) \\
& =p q t+\frac{p q(q-1)}{2}\left(n \sum_{i=1}^{m} r_{i}-\sum_{i=1}^{m} r_{i}^{2}\right) \\
& =p q t+\frac{p q(q-1)}{2}\left(n \sum_{i=1}^{m} u_{i}-\sum_{i=1}^{m} u_{i}^{2}\right) \\
& =p q t+\frac{p q(q-1)}{2} \sum_{i=1}^{m} u_{i} \cdot\left(n-u_{i}\right)=\beta .
\end{aligned}
$$

This proves that $A^{(p \times q)}$ and $B^{(p \times q)}$ are $M$-row equivalent.

Remark 1. The sufficient condition in Theorem 3 is not vacuous as can be seen from the following illustration.

Consider the binary arrays $A=\begin{array}{llll}a & a & b & b \\ a & b & a & a\end{array}$ and $B=$ $\begin{array}{llll}a & a & a & b \\ b & a & a & b\end{array}$ which are $M$-equivalent with the row Parikh matrix $\left(\begin{array}{lll}1 & 5 & 5 \\ 0 & 1 & 3 \\ 0 & 0 & 1\end{array}\right)$. If the number of subword $a$ in the rows of $A$ (resp. $B$ ) are $r_{1}$ and $r_{2}$ (resp. $u_{1}$ and $u_{2}$ ), then $r_{1}^{2}+r_{2}^{2}=u_{1}^{2}+u_{2}^{2}=13$. Now, $\quad A^{(3 \times 2)}=$

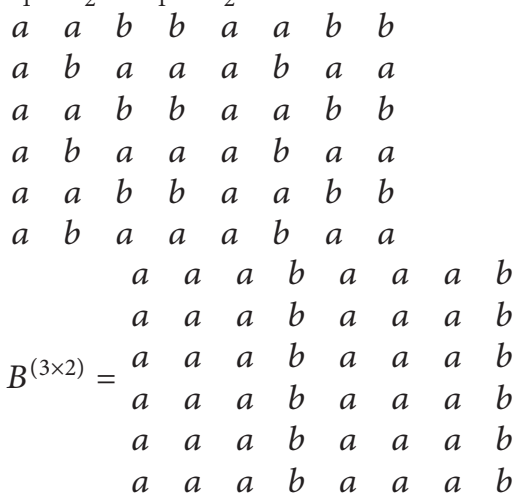
and $M_{r}(A)=M_{r}(B)=\left(\begin{array}{ccc}1 & 30 & 51 \\ 0 & 1 & 18 \\ 0 & 0 & 1\end{array}\right)$ so that the binary arrays $A^{(3 \times 2)}$ and $B^{(3 \times 2)}$ are $M$-equivalent.

\section{Fair Picture Arrays}

Fair words and their properties have been studied in [24]. A weak ratio property for an array is introduced in [21]. We now extend the notion of fair words to two-dimensional arrays. We also recall the notion of the weak ratio property restricting it to binary arrays.

\section{Definition 5}

(i) A binary array $A \in \Sigma^{m \times n}$ is called fair if the total number of subwords $a b$ in the rows (respectively, columns) of $A$ is equal to the total numbers of subwords $b a$ in the rows (respectively, columns) of $A$

(ii) Let $A$ and $B$ be two binary arrays over $\Sigma=\{a<b\}$. The arrays $A$ and $B$ are said to satisfy a weak ratio property if $\left(|A|_{a} /|B|_{a}\right)=\left(|A|_{b} /|B|_{b}\right)=k$ where $k$ is a nonzero constant

Theorem 4. Let $A$ and $B$ be two fair binary arrays over $\Sigma=\{a<b\}$, both having the same number of rows, satisfying the weak ratio property. Then, the arrays $A \circ B$ and $B \circ A$ are also fair. A corresponding result holds good for $A \diamond B$ and $B \diamond A$.

Proof. Let $A \in \sum^{m \times n}$ and $B \in \sum^{m \times l}$ be two fair words satisfying the weak ratio property with ratio constant $\alpha$. Denoting the total number of subwords $a b$ in the rows of a binary array by $|X|_{a b}^{r}$, we have $|A|_{a b}^{r}=|A|_{b a}^{r}$ and $|B|_{a b}^{r}=|B|_{b a}^{r}$. Also, we have $\left(|A|_{a} /|B|_{a}\right)=\left(|A|_{b} /|B|_{b}\right)=\alpha$. This implies that $m n=|A|=|A|_{a}+|A|_{b}=\alpha\left(|B|_{a}+|B|_{b}\right)=\alpha m l$, i.e., $n=\alpha l$.

Since $A \circ B$ is the column catenation of $A$ and $B$, the column Parikh matrix of $A \circ B$ is $M_{c}(A \circ B)=M_{c}(A) \oplus$ $M_{c}(B)$. Therefore, the number of subword $a b$ 's column wise in $A \circ B$ is the same as the number of $b a$ 's column wise in $A \circ B$.

Let $x_{i}$ and $u_{i}, 1 \leq i \leq m$, be the words in the $i^{\text {th }}$ row of $A$ and $B$, respectively. Now, the number of $a b$ 's row wise in $A \circ B$ is given by

$$
\begin{aligned}
|A \circ B|_{a b}^{r} & =\sum_{i=1}^{m}\left(\left|x_{i}\right|_{a b}+\left|y_{i}\right|_{a b}+\left|x_{i}\right|_{a} \cdot\left|y_{i}\right|_{b}\right) \\
& =|A|_{a b}^{r}+|B|_{a b}^{r}+\sum_{i=1}^{m}\left|x_{i}\right|_{a} \cdot\left|y_{i}\right|_{b} .
\end{aligned}
$$

We also have

$$
\begin{aligned}
|A \circ B|_{b a}^{r} & =\sum_{i=1}^{m}\left(\left|x_{i}\right|_{b a}+\left|x_{i}\right|_{b a}+\left|x_{i}\right|_{b} \cdot\left|y_{i}\right|_{a}\right) \\
& =|A|_{b a}^{r}+|B|_{b a}^{r}+\sum_{i=1}^{m}\left|x_{i}\right|_{b} \cdot\left|y_{i}\right|_{a} \\
& =|A|_{a b}^{r}+|B|_{a b}^{r}+\sum_{i=1}^{m}\left|x_{i}\right|_{b}\left(l-\left|y_{i}\right|_{b}\right),
\end{aligned}
$$

since 


$$
\begin{aligned}
\left|y_{i}\right| & =l \\
& =|A|_{a b}^{r}+|B|_{a b}^{r}+l \sum_{i=1}^{m}\left|x_{i}\right|_{b}-\sum_{i=1}^{m}\left|x_{i}\right|_{b}\left|y_{i}\right|_{b} \\
& =|A|_{a b}^{r}+|B|_{a b}^{r}+\alpha l \sum_{i=1}^{m}\left|y_{i}\right|_{b}-\sum_{i=1}^{m}\left|x_{i}\right|_{b}\left|y_{i}\right|_{b} \\
& =|A|_{a b}^{r}+|B|_{a b}^{r}+\sum_{i=1}^{m}\left(\alpha l-\left|x_{i}\right|_{b}\right)\left|y_{i}\right|_{b} \\
& =|A|_{a b}^{r}+|B|_{a b}^{r}+\sum_{i=1}^{m}\left(n-\left|x_{i}\right|_{b}\right)\left|y_{i}\right|_{b},
\end{aligned}
$$

since

$$
\begin{aligned}
n & =\alpha l \\
& =|A|_{a b}^{r}+|B|_{a b}^{r}+\sum_{i=1}^{m}\left|x_{i}\right|_{a} \cdot\left|y_{i}\right|_{b}=|A \circ B|_{a b}^{r} .
\end{aligned}
$$

This proves that $A \circ B$ is a fair array. In a similar manner, it can be shown that $B \circ A$ is also a fair array.

\section{Restricted Shuffle Operator on Picture Arrays}

In [6], a restricted shuffle operator on two binary words, denoted as SShuf, is considered and properties of Parikh matrices of words under this operator are derived, especially over a binary alphabet. Here, we extend this operator to picture arrays and obtain properties of Parikh matrices of arrays under this operator.

Definition 6. Let $A, B \in \sum^{m \times n}$ be two picture arrays over $\Sigma=$ $\{a<b\} \quad$ such that $A=\begin{array}{ccc}a_{11} & \cdots & a_{1 n} \\ \vdots & \ddots & \vdots \\ a_{m 1} & \cdots & a_{m n}\end{array}$ and $B=\begin{array}{ccc}b_{11} & \cdots & b_{1 n} \\ \vdots & \ddots & \vdots \\ b_{m 1} & \cdots & b_{m n}\end{array}$. Then, the restricted row shuffle operator on the pair of arrays $A$ and $B$ is defined by

$$
\begin{aligned}
& \begin{array}{lllllll}
a_{11} & b_{11} & a_{12} & b_{12} & \cdots & a_{1 n} & b_{1 n}
\end{array} \\
& \begin{array}{lllllll}
a_{21} & b_{21} & a_{22} & b_{22} & \cdots & a_{2 n} & b_{2 n}
\end{array} \\
& \operatorname{RSShuf}(A, B)= \\
& \begin{array}{lllllll}
a_{m 1} & b_{m 1} & a_{m 2} & b_{m 2} & \cdots & a_{m n} & b_{m n}
\end{array}
\end{aligned}
$$

and similarly the restricted column shuffle operator is defined by

$$
\begin{array}{ccccc}
a_{11} & a_{12} & \cdots & a_{1 n} \\
b_{11} & b_{12} & \cdots & b_{1 n} \\
\cdot & \cdot & \cdot & \cdot \\
\operatorname{CSShuf}(A, B)= & \cdot & \cdot & \cdot & \cdot \\
\cdot & \cdot & \cdot & \cdot \\
a_{m 1} & a_{m 2} & \cdots & a_{m n} \\
b_{m 1} & b_{m 2} & \cdots & b_{m n}
\end{array}
$$

Example 3. Let $A, B \in \Sigma^{3 \times 3}$ over the binary alphabet $\Sigma=$ $\{a<b\}$ be given by $A=\begin{array}{lll}a & a & b \\ b & a & a\end{array}, B=\begin{array}{lll}b & a & a \\ a & b & a\end{array}$. Then, $\operatorname{RSShuf}(A, B)=\begin{array}{llllll}a & b & a & a & b & a \\ b & a & a & b & a & a\end{array} \quad$ and

$\operatorname{CSShuf}(A, B)=\begin{array}{lll}a & a & b \\ b & a & a \\ b & a & a \\ a & b & a\end{array}$

We observe a few facts which are immediate from the definition:

(i) $M_{r}(\operatorname{CSShuf}(A, B))=M_{r}(A) \oplus M_{r}(B)$

(ii) $M_{c}(\operatorname{RSShuf}(A, B))=M_{c}(A) \oplus M_{c}(B)$

In [7], the authors introduced a notion of the positions of letters in a word and using this notion characterized the $M$-equivalent words over the binary alphabet. The sum of positions of a letter $a$ in a word $w$ of length $n$ over an alphabet $\Sigma_{k}$, denoted by $S_{a}(w)$, is defined by $S_{a}(w)=\sum_{w[i]=a, 1 \leq i \leq n} i$.

Here, we introduce the sum of positions of a letter in a binary array over $\Sigma=\{a<b\}$ as follows.

Definition 7. Let $A$ be a binary $m \times n$ array over $\{a<b\}$, then the row-wise sum of positions of a letter $a$ in $A$ is defined by $S_{a}^{r}(A)=\sum_{1 \leq i \leq m} S_{a}\left(x_{i}\right)$, where $x_{i}$ is the $i^{\text {th }}$ row of the array $A$. Similarly, the column-wise sum of positions of a letter $a$ in $A$ is defined by $S_{a}^{c}(A)=\sum_{1 \leq i \leq n} S_{a}\left(y_{i}\right)$, where $y_{i}$ is the $i^{\text {th }}$ column of the array $A$.

Theorem 5. Two arrays $A$ and $B$ over $\{a<b\}$ is $M$-row equivalent (column equivalent) to each other if each row (column) of $A$ and $B$ has the same number of b's and $S_{b}^{r}(A)=$ $S_{b}^{r}(B)\left(S_{b}^{c}(A)=S_{b}^{c}(B)\right.$, respectively).

Proof. Let $x_{i}$ and $y_{i}$ be the $i^{\text {th }}$ row of the arrays $A$ and $B$, respectively. Also, let $\left|x_{i}\right|_{b}=\left|y_{i}\right|_{b}$, for all $1 \leq i \leq m$, and $S_{b}^{r}(A)=S_{b}^{r}(B)$. Then, the number of $a b$ 's in the row Parikh matrix of $A$ is equal to $\sum_{1 \leq i \leq m}\left|x_{i}\right|_{a b}$. Now, 


$$
\begin{aligned}
\sum_{1 \leq i \leq m}\left|x_{i}\right|_{a b} & =\sum_{1 \leq i \leq m}\left[S_{b}\left(x_{i}\right)-\frac{\left|x_{i}\right|_{b}\left(\left|x_{i}\right|_{b}+1\right)}{2}\right] \\
& =\sum_{1 \leq i \leq m} S_{b}\left(x_{i}\right)-\sum_{1 \leq i \leq m} \frac{\left|x_{i}\right|_{b}\left(\left|x_{i}\right|_{b}+1\right)}{2} \\
& =\sum_{1 \leq i \leq m} S_{b}\left(y_{i}\right)- \\
\sum_{1 \leq i \leq m} \frac{\left|y_{i}\right|_{b}\left(\left|y_{i}\right|_{b}+1\right)}{2} & =\sum_{1 \leq i \leq m}\left[S_{b}\left(y_{i}\right)-\frac{\left|y_{i}\right|_{b}\left(\left|y_{i}\right|_{b}+1\right)}{2}\right] \\
& =\sum_{1 \leq i \leq m}\left|y_{i}\right|_{a b},
\end{aligned}
$$

which is the number of $a b$ 's in the row Parikh matrix of $B$. Hence, the binary arrays $A$ and $B$ are $M$-row equivalent.

Similarly, the other case of $M$-column equivalence can be proved.

Lemma 1. Let $A, B \in \Sigma^{m \times n}$ where $\Sigma=\{a<b\}$, then (i) $S_{b}^{r}(\operatorname{RSShuf}(A, B))=2\left(S_{b}^{r}(A)+S_{b}^{r}(B)\right)-|A|_{b} \quad$ and (ii) $S_{b}^{r}(\operatorname{CSShuf}(A, B))=2\left(S_{b}^{c}(A)+S_{b}^{c}(B)\right)-|A|_{b}$ where $|A|_{b}$ is the number of $b$ 's in the array $A$.

Proof. Let $x_{i}$ and $y_{i}$ be the $i^{\text {th }}$ row of the arrays $A$ and $B$, respectively. Then, we have

$$
\begin{aligned}
S_{b}^{r}(\operatorname{RSShuf}(A, B)) & =\sum_{i=1}^{m} S_{b}\left(\operatorname{SShuf}\left(x_{i}, y_{i}\right)\right) \\
& =\sum_{i=1}^{m}\left[2\left\{S_{b}\left(x_{i}\right)+S_{b}\left(y_{i}\right)\right\}-\left|x_{i}\right|_{b}\right] \\
& =2\left[\sum_{i=1}^{m} S_{b}\left(x_{i}\right)+\sum_{i=1}^{m} S_{b}\left(y_{i}\right)\right]-\sum_{i=1}^{m}\left|x_{i}\right|_{b} \\
& =2\left(S_{b}^{r}(A)+S_{b}^{r}(B)\right)-|A|_{b} .
\end{aligned}
$$

Similarly, we can prove the statement (ii). A sufficient condition for the row shuffle operator of two binary arrays is given as follows.

Theorem 6. Let $A, B \in \Sigma^{m \times n}$ where $\Sigma=\{a<b\}$, then $\operatorname{RSShuf}(A, B) \equiv{ }_{M} \operatorname{RSShuf}(B, A)$ if $|A|_{b}=|B|_{b}$.

This can be seen using Lemma 1 and the fact that $M_{c}(\operatorname{RSShuf}(A, B))=M_{c}(A) \oplus M_{c}(B)$.

\section{Geometric Operations on Picture Arrays}

Geometric operations on picture arrays such as reflection and rotation are now considered. Properties of Parikh matrices of the arrays resulting from the geometric operations are obtained.
Proposition 2. Let $A$ be a binary $m \times n$ picture array over $\{a<b\}$. Reflection of $A$ about its rightmost vertical yields an array $A_{v}$ with the following properties:

(i) $\left|A_{v}\right|_{a}=|A|_{a}$ and $\left|A_{v}\right|_{b}=|A|_{b}$

(ii) The column Parikh matrices of $A$ and $A_{v}$ are the same

(iii) The number of ab's row wise in $A_{v}$ is $\left|A_{\nu}\right|_{a b}^{r}=\sum_{1<i<m}\left(\left|r_{i}\right|_{a}\left|r_{i}\right|_{b}-\left|r_{i}\right|_{a b}\right)$, where $r_{i}$ is the $i^{\text {th }}$ row of $A$

Similarly, reflection of A about its bottommost horizontal yields an array $A_{h}$ with the following properties:

(i) $\left|A_{h}\right|_{a}=|A|_{a}$ and $\left|A_{h}\right|_{b}=|A|_{b}$

(ii) The row Parikh matrices of $A$ and $A_{h}$ are the same

(iii) The number of ab's column wise in $A_{h}$ is $\left|A_{h}\right|_{a b}^{c}=\sum_{1 \leq i \leq n}\left(\left|c_{i}\right|_{a}\left|c_{i}\right|_{b}-\left|c_{i}\right|_{a b}\right)$, where $c_{i}$ is the $i^{\text {th }}$ column of $A$

The following proposition is a consequence of Proposition 2.

Proposition 3. If two arrays $A$ and $B$ of the same sizes are $M$-equivalent, then their reflections about their rightmost verticals and their bottommost horizontals are also M-equivalent.

Definition 8. Let $A \in \sum^{m \times n}$ be a picture array over $\Sigma=\{a<b\}$ such that $A=\begin{array}{ccc}a_{11} & \cdots & a_{1 n} \\ \vdots & \ddots & \vdots\end{array}$. A picture array obtained from $A$ by rotating it by $90^{\circ}$ clockwise, denoted by $A^{90^{\circ}}$, is defined as $A^{90^{\circ}}=\begin{array}{ccc}a_{m 1} & \cdots & a_{11} \\ \vdots & \ddots & \vdots \\ a_{m n} & \cdots & a_{1 n}\end{array}$.

Note that $A^{90^{\circ}}$ is an array of size $n \times m$ such that the first row of $A$ is the last column of $A^{90^{\circ}}$, the second row of $A$ is the last but the second column of $A^{90^{\circ}}$, and so on, and the last row of $A$ is the first column of $A^{90^{\circ}}$.

Similarly, one can define $A^{180^{\circ}}$ (which is the same as $\left.\left(A^{90^{\circ}}\right)^{90^{\circ}}\right), A^{270^{\circ}}$, and $A^{360^{\circ}}$. It is easy to see that $A^{360^{\circ}}=A$.

Now we state in the following proposition, the relations between the row and column Parikh matrices of the rotated arrays.

Proposition 4. Let $A \in \Sigma^{m \times n}$ be a picture array where $\Sigma=\{a<b\}$, then (i) $M_{r}\left(A^{90}\right)=M_{c}\left(A_{h}\right)$ and $M_{c}\left(A^{90}\right)=M_{r}(A), \quad$ (ii) $M_{r}\left(A^{180}\right)=M_{r}\left(A_{v}\right) \quad$ and $M_{c}\left(A^{180^{\circ}}\right)=M_{c}\left(A_{v}\right)$, and (iii) $M_{r}\left(A^{270}\right)=M_{c}(A)$ and $M_{c}\left(A^{270^{\circ}}\right)=M_{r}\left(A_{v}\right)$ where $A_{h}$ and $A_{v}$ are the reflections of the array $A$ about its bottommost horizontal and rightmost vertical.

\section{Concluding Remarks}

Motivated by applications in areas such as pattern recognition and computer vision several studies have been done on combinatorial properties of two-dimensional arrays [15]. The study done in this paper is a contribution to this area as well, and it extends notions and concepts well studied in the 
context of strings. It will be of interest to consider picture arrays of three or more symbols and examine the applicability of the notions and results considered here.

\section{Data Availability}

The data related to the findings of this work are available in journal articles.

\section{Conflicts of Interest}

The authors declare that there are no conflicts of interest regarding the publication of this article.

\section{Acknowledgments}

S. Bera and L. Pan were partially supported by the National Natural Science Foundation of China (61772214).

\section{References}

[1] M. Lothaire, Combinatorics on Words, Cambridge Mathematical Library, Cambridge University Press, Cambridge, UK, 1997.

[2] J. Berstel and D. Perrin, "The origins of combinatorics on words," European Journal of Combinatorics, vol. 28, no. 3, pp. 996-1022, 2007.

[3] G. Rozenberg and A. Salomaa, Handbook of Formal Languages, vol. 1-3, Springer-Verlag, New York, NY, USA, 1997.

[4] A. Mateescu, A. Salomaa, K. Salomaa, and S. Yu, "A sharpening of the Parikh mapping," RAIRO-Theoretical Informatics and Applications, vol. 35, no. 6, pp. 551-564, 2001.

[5] A. Atanasiu, "Binary amiable words," International Journal of Foundations of Computer Science, vol. 18, no. 2, pp. 387-400, 2007.

[6] A. Atanasiu and W. C. Teh, "A new operator over Parikh languages," International Journal of Foundations of Computer Science, vol. 27, no. 6, pp. 757-769, 2016.

[7] S. Fossé and G. Richomme, "Some characterizations of Parikh matrix equivalent binary words," Information Processing Letters, vol. 92, no. 2, pp. 77-82, 2004.

[8] A. Mateescu, "Algebraic aspects of Parikh matrices," in Theory is Forever, pp. 170-180, Springer, Berlin, Germany, 2004.

[9] K. G. Subramanian, A. M. Huey, and A. K. Nagar, "On Parikh matrices," International Journal of Foundations of Computer Science, vol. 20, no. 2, pp. 211-219, 2009.

[10] W. C. Teh, "On core words and the Parikh matrix mapping," International Journal of Foundations of Computer Science, vol. 26, no. 1, pp. 123-142, 2015.

[11] W. C. Teh and K. H. Kwa, "Core words and Parikh matrices," Theoretical Computer Science, vol. 582, pp. 60-69, 2015.

[12] A. Salomaa, "Comparing subword occurrences in binary D0L sequences," International Journal of Foundations of Computer Science, vol. 18, no. 6, pp. 1395-1406, 2007.

[13] R.-F. Atanasiu, "Message authentication code based on Parikh matrices," in Proceedings of the International Conference on Security for Information Technology and Communication, pp. 7-14, Bucharest, Romania, 2008.

[14] A. Amir and G. Benson, "Two-dimensional periodicity in rectangular arrays," SIAM Journal on Computing, vol. 27, no. 1, pp. 90-106, 1998.

[15] A. Amir, G. M. Landau, S. Marcus, and D. Sokol, "Two-dimensional maximal repetitions," in Proceedings of the LIPIcs-
Leibniz International Proceedings in Informatics 112, 26th Annual European Symposium on Algorithms (ESA), vol. 2, no. 1-2, p. 14, Helsinki, Finland, August 2018.

[16] A. Apostolico and V. E. Brimkov, "Fibonacci arrays and their two-dimensional repetitions," Theoretical Computer Science, vol. 237, no. 1-2, pp. 263-273, 2000.

[17] A. Carpi and A. de Luca, "Repetitions and boxes in words and pictures," Jewels are Forever, pp. 295-306, 1999.

[18] A. Carpi and A. de Luca, "Periodic-like words, periodicity, and boxes," Acta Informatica, vol. 37, no. 8, pp. 597-618, 2001.

[19] A. Carpi and A. de Luca, "Repetitions, fullness, and uniformity in two-dimensional words," International Journal of Foundations of Computer Science, vol. 15, no. 2, pp. 355-383, 2004.

[20] G. Gamard, G. Richomme, J. Shallit, and T. J. Smith, "Periodicity in rectangular arrays," Information Processing Letters, vol. 118, pp. 58-63, 2017.

[21] K. G. Subramanian, K. Mahalingam, R. Abdullah, and A. K. Nagar, "Two-dimensional digitized picture arrays and Parikh matrices," International Journal of Foundations of Computer Science, vol. 24, no. 3, pp. 393-408, 2013.

[22] V. Masilamani, K. Krithivasan, K. G. Subramanian, and A. M. Huey, "Efficient algorithms for reconstruction of 2Darrays from extended Parikh images," in ISVC 2008 Part II, Lecture Notes in Computer Science, G. Bebis, Ed., vol. 5359, pp. 1137-1146, Springer, Berlin, Germany, 2008.

[23] A. Atanasiu, G. Poovanandran, and W. C. Teh, "Parikh matrices for powers of words," Acta Informatica, vol. 56, no. 6, pp. 521-535, 2019.

[24] A. Cerny, "On fair words," Journal of Automata, Languages, and Combinatorics, vol. 14, no. 2, pp. 163-174, 2009.

[25] S. Bera, A. K. Nagar, L. Pan, S. Sriram, and K. G. Subramanian, "Parikh matrices of binary picture arrays," in Proceedings of the MICOPAM 2018 International Conference, pp. 157-161, Antalya, Turkey, October 2018. 